\title{
The Duke Employee Weight Loss Program: Report from a Duke Diet and Fitness Center Pilot Study
}

\begin{abstract}
:
Background: Obesity is increasingly prevalent in the industrialized world. Obese workers have two times the number of workers' compensation claims as those of non-obese workers. Worksite interventions may be especially effective because employees spend a large part of their day in the work environment, and both employee and employer have incentives to improve the employees' health. Objective: To assess the impact of a 4 week employee intervention in participant's weight, body fat and blood pressure. Methods: This was a pilot study. We assessed feasibility and impact compared to baseline at 4 weeks, 6 months, and 12 months post-program. The primary outcomes were weight and body mass index. Secondary outcomes included percent body fat and blood pressure. Results: Forty participants consented to be part of the study. Baseline weight was $97.8 \mathrm{~kg}$ [SD \pm 17.05$]$. Twelve months post-intervention weight loss averaged $2.7 \mathrm{~kg}$ [SD \pm 6.35$]$. One-way repeated measures ANOVA showed a significant effect of participation on weight, body mass index, and percent body fat. Conclusion: Significant improvements in the primary outcomes were observed in participants completing our worksite pilot study, indicating that a worksite weight loss intervention is potentially effective.
\end{abstract}

Key Words: Obesity, worksite, intervention, employee.

\section{Introduction}

Obesity is increasingly prevalent in the industrialized world, with serious implications for health and healthcare costs $[1,2]$. Obese workers have two times the number of workers' compensation claims as those of non-obese workers[3]. In addition, estimates of medical expenditures attributable to overweight and obesity range from $\$ 170$ per year for overweight male employees to $>\$ 1500$ per year for obese female employees[4]. Worksite interventions may be especially effective because employees spend a large part of their day in the work environment, and both employee and employer have incentives to improve the employees' health. Indeed, such interventions have become more prevalent over the past decade [1, 5-9]. However, the literature reporting on these important programs is rife with methodological problems $[10,11]$ that must be systematically addressed; outcomes for worksite interventions must be rigorously measured. A necessary first step in the process of conducting a large scale trial is to establish the feasibility of rigorously measuring outcomes in existing worksite weight loss programs.

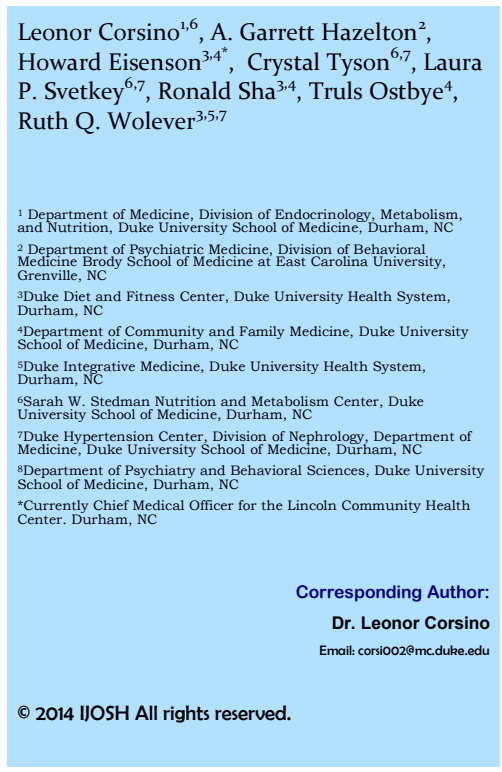

The design of the Duke Employee Weight Loss (DEWL) program allows us to address methodological shortcomings of prior worksite weight management studies. Specifically, funding constraints and competing employer needs typically undermine ability to adhere to rigorous methodology in assessing clinical programs in the worksite. In fact, pragmatic program evaluation does not typically approximate the rigor of federally-funded outcome trials.

The purpose of this study was to test the feasibility and efficacy of the DEWLP using rigorous measurement methods to set the stage for a large randomized trial of the unique DEWL program.

\section{Methods}

Intervention- Duke Employee Weight Loss Program

The DEWL program was modeled on the long-established Duke Diet and Fitness Center (DFC) "immersion" approach to weight loss and improved fitness but adapted to accommodate the schedules of working individuals. The original DFC immersion approach provides all meals, daily exercise classes, and didactic sessions to participants who are "in residence" daily, for periods 
of time ranging from one week to several months, but most commonly for four weeks. [12, 13]

In essence, the DEWL program repackaged critical elements of the DFC residential program to create a "mini-immersion" program for busy working adults. Specially, the DEWLP program was a 4 week program delivered to 4 cohorts of participants at the Duke Diet and Fitness Center ( DFC)facility located on the Duke University Health System space. This "mini- immersion" program approached lifestyle changes based on the Diet and Fitness center and other residential programs $[12,13]$ that operate on the theory that regular practice of healthful habits of eating and exercise, coupled with skill-building classes and a supportive peer group milieu, facilitate learning new habits and yield meaningful short-term results that enhance confidence and motivation[12-16]. During the program, participants were offered a total of 40 meals (10 per week) prepared by the DFC culinary staff using recipes developed for the residential program. Entrees were designed to be compatible with a balanced and calorie controlled diet (approximately 40-50\% of the calories from carbohydrates, $20-25 \%$ from protein, $20-25 \%$ from fat) that would typically represent $1100-1300$ calories per day for females and 1400-1600 calories per day for males. In addition, participants were offered 20 group exercise sessions (5 per week) led by a fitness specialist or exercise physiologist, and 20 group educational sessions (5 per week) led by dieticians, exercise physiologists, psychologists/clinical social workers, and physicians. Further, participants were offered the chance to exercise independently during additional hours of operation of the facility (7:30 AM to 9:00 PM Monday through Friday, and 8:00 AM to 9:00 PM Saturday and Sunday). The structure of the program required a two hour commitment each Monday thru Thursday evening, and Saturday morning; a total of 10 hours a week. Participants received a 1 hour fitness class (e.g. cardio blast, aqua aerobics, Latin dance) followed by a meal (dinner on weeknights, lunch on Sat) which was provided during a 45 minutes educational session (the remaining 5 of their 10 weekly meals could be consumed in the facility, at other mealtimes or taken out). The educational session topics included diet and exercise space to support weight loss and optimal health, as well as cognitive behavioral strategies to support sustainable lifestyle change including self-awareness, goal setting, overcoming obstacles, stress management, and emotion regular. After completing the 4 week program, participants had the opportunity to purchase additional monthly membership at the Center (for a fee of \$125), which included 10 meals per week and full access to the DFC facilities. Additional meals were available for purchase a la carte.

\section{Study population and methods}

Using the DEWL program cohorts that were enrolled between October of 2009 and March 2012, a total of 40 participants enrolled in the pilot study. Participants included were overweight or obese (BMI $\geq 25 \mathrm{~kg} / \mathrm{m} 2$ ), adult (> 18 years), Duke employees and their spouses, same-sex partners, and children (over the age of 18) who were enrolled in the DEWL program. Participants for the study were recruited from four successive cohorts of the DEWL program.

Participants were recruited through one of two "opt out" procedures. First, those enrolled in the DEWL program who attended an informational pre-program session were told about the study and invited to participate in the measurement study. Second, DEWL program participants who did not attend an informational session were contacted by study staff and given the opportunity to opt out if not interested in taking part in the study. Although DEWL program participants were paying $\$ 650$ for the program itself, there was no additional cost to study participants who volunteered to provide data. Benefits offered to study participants included receiving, at the end of the study, a copy of all measurements taken as part of the study. In addition, there was a modest incentive for participation, with eight meal vouchers for the Duke Diet and Fitness Center (DFC) provided to those participants who completed the 12 months post intervention data collection. All participants received clearance from their primary care provider or the DFC medical staff prior to enrollment into the DEWL program. All aspects of the study were approved by the Duke Institutional Review Board, and all participants provided written informed consent.

We conducted an observational, prospective pilot study that allowed each participant to serve as his/her own control, with four measurement time points: baseline, 4 weeks, 6 months post-program, and 12 months post-program. Feasibility was assessed by percentage of DEWLP that agreed to participate in the study, percentage that completed the study, and percentage with data collection at all-time points. The primary outcome to assess effectiveness of the study was change in weight and BMI. Secondary outcomes included change in percentage body weight and systolic and diastolic blood pressure.

\section{Measurements}

All measurements were obtained by trained and certified research personnel using methods from multicenter randomized controlled trials $[17,18]$. Data collection visits were conducted at baseline, at the end of the 4 week program, and at 6 and 12 months post-program. Weight and percentage body fat were measured at each time point using a calibrated digital scale (Tanita, model \# TBF-310GF- Tanita, Arlington Heights, Illinois) with the participant wearing a standardized outfit of light, indoor clothes without shoes. Percentage body fat was estimated by the Tanita scale using bioelectrical impedance assay. Height was measured at baseline and 12 months post -program using a wall-mounted stadiometer. Body mass index was calculated using the Quetelet Index, (weight [kg]/height [m2]). Arm circumference was measured at each time point using a metric tape measure to determine proper cuff size for blood pressure. Participants were asked to refrain from eating, smoking, and exercising for at least 30 minutes prior to blood pressure measurement. After resting in seated position for 5 minutes in a quiet room free from activity, three consecutive blood pressure measurements were taken 1 minute apart and averaged using 
an automated oscillometric device (Omron HEM-907XL OMRON Healthcare, Bannockburn, Illinois). In addition, demographic information was obtained through a short self- administered questionnaire.

\section{Statistical methods}

For feasibility measures, descriptive statistics were provided. While this pilot study was not powered for definitive hypothesis testing, we wished to estimate the effect size for future trials. Hence, for our primary outcomes, one-way repeated measures ANOVA procedures were conducted to assess the effect of the DEWL program on weight and BMI across four time points (i.e., baseline, 4 weeks, 6 and 12 months post-program). Paired samples t-tests were then conducted post-hoc to clarify the simple effects when a main effect for time was discovered. Similar procedures were conducted to assess secondary outcomes including percent body fat and blood pressure (systolic and diastolic).

For each impact measure, we set an overall alpha level of 0.05 for the main effect, and similarly set the alpha at 0.05 for posthoc comparisons. All analyses were carried out using IMB SPSS Statistics, Version 19.

\section{Results}

Participants enrolled in the study had an average age of 48.2 (SD \pm 10.7 ) years, $88 \%$ were female, $70 \%$ white, $30 \%$ African American, and $3 \%$ Hispanic. Fifty-five percent were married. All participants had at least a high school education. Ninety eight percent were employed full-time and $65 \%$ had an annual household income of $>\$ 60,000$. At baseline, none of the participants were current smokers, though $33 \%$ reported a prior history of smoking. The difference between enrolled and those that completed the 12 months data collection are included in Table I.

\section{Feasibility}

Of the possible pool of 55 participants who were participating in the DEWL program, 40 (73\%) consented and enrolled to undergo measurements for this study. Of the 40 that enrolled, 38 had primary outcome data collected at baseline. Of the $15(27 \%)$ who did not participate in the study, seven opted out due to low interest; three were not eligible because of a $\mathrm{BMI}<25$; four reported schedule conflicts as the reason for non-participation, and one was excluded by the investigators. The participant had previously lost a significant amount of weight with the program and may have skewed the impact results (Fig 1).

In terms of retention, 27 (68\%) participants completed the 6 months post program follow-up assessment, 22 (55\%) completed data collection at all times points and 29 (73\%) participants completed the study, including the 12 months post program follow-up visit. Comparison of baseline characteristics between those who completed the data collection at 12 months post intervention and the non-completers (data not shown) only revealed 1 statistically significant difference, with completers
Table I Participants baseline characteristics

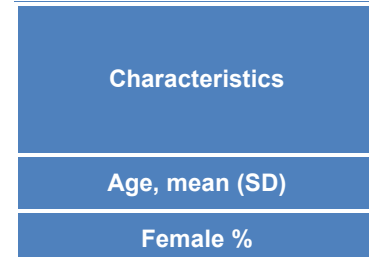

Female \%

Race/ethnicity \%

African American

White

Hispanic

Marital status \%

Married

Single

Cohabitating

Divorced

Separated

Widowed

\begin{tabular}{|c|}
\hline Married \\
\hline Single \\
\hline Cohabitating \\
\hline Divorced \\
\hline Separated \\
\hline Widowed \\
\hline
\end{tabular}

Living arrangement $\%$

Alone

Partner/spouse

Partner / spouse \& children

Parent/other relatives

Other

Education \%

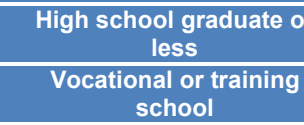

Some college

College graduate

Some post graduate

Masters

Doctorate

Employment \%

Full-time

Part-time

Annual household income, $\$ \%$

$<30,000$

$30,000-60,000$

$>60,000$

Select Medical

Characteristics \%

Current cigarette smokers

Overweight

(BMI 25-29.9), \%

Obese (BMI $\geq 30$ ), \%

Hypertension, $\%$

Diabetes, $\%$

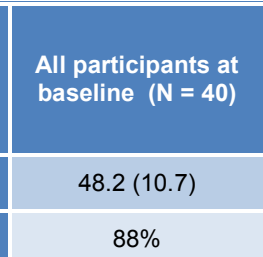

Participants with

baseline and 12 -

month data $(\mathrm{N}=29)$

$88 \%$

$48.0(10.0)$

$30 \%$

$28 \%$

$70 \%$

$3 \%$

$66 \%$

$3 \%$

\section{$55 \%$}

\begin{tabular}{|c|c|}
\hline $\begin{array}{l}\text { All participants at } \\
\text { baseline }(N=40)\end{array}$ & $\begin{array}{c}\text { Participants with } \\
\text { baseline and } 12- \\
\text { month data }(\mathbf{N}=29)\end{array}$ \\
\hline $48.2(10.7)$ & $48.0(10.0)$ \\
\hline $88 \%$ & $90 \%$ \\
\hline $30 \%$ & $28 \%$ \\
\hline $70 \%$ & $66 \%$ \\
\hline $3 \%$ & $3 \%$ \\
\hline $55 \%$ & $48 \%$ \\
\hline $18 \%$ & $24 \%$ \\
\hline $5 \%$ & $3 \%$ \\
\hline $18 \%$ & $17 \%$ \\
\hline $3 \%$ & $3 \%$ \\
\hline $3 \%$ & $3 \%$ \\
\hline $33 \%$ & $41 \%$ \\
\hline $33 \%$ & $31 \%$ \\
\hline $30 \%$ & $24 \%$ \\
\hline $3 \%$ & $3 \%$ \\
\hline $3 \%$ & $3 \%$ \\
\hline $0 \%$ & $0 \%$ \\
\hline $3 \%$ & $3 \%$ \\
\hline $15 \%$ & $17 \%$ \\
\hline $28 \%$ & $27 \%$ \\
\hline $5 \%$ & $3 \%$ \\
\hline $35 \%$ & $31 \%$ \\
\hline $15 \%$ & $17 \%$ \\
\hline $98 \%$ & $100 \%$ \\
\hline $3 \%$ & $0 \%$ \\
\hline $3 \%$ & $0 \%$ \\
\hline $30 \%$ & $34 \%$ \\
\hline
\end{tabular}

$5 \%$

\begin{tabular}{|c|c|}
\hline $\begin{array}{l}\text { All participants at } \\
\text { baseline }(N=40)\end{array}$ & $\begin{array}{c}\text { Participants with } \\
\text { baseline and } 12- \\
\text { month data }(\mathbf{N}=29)\end{array}$ \\
\hline $48.2(10.7)$ & $48.0(10.0)$ \\
\hline $88 \%$ & $90 \%$ \\
\hline $30 \%$ & $28 \%$ \\
\hline $70 \%$ & $66 \%$ \\
\hline $3 \%$ & $3 \%$ \\
\hline $55 \%$ & $48 \%$ \\
\hline $18 \%$ & $24 \%$ \\
\hline $5 \%$ & $3 \%$ \\
\hline $18 \%$ & $17 \%$ \\
\hline $3 \%$ & $3 \%$ \\
\hline $3 \%$ & $3 \%$ \\
\hline $33 \%$ & $41 \%$ \\
\hline $33 \%$ & $31 \%$ \\
\hline $30 \%$ & $24 \%$ \\
\hline $3 \%$ & $3 \%$ \\
\hline $3 \%$ & $3 \%$ \\
\hline $0 \%$ & $0 \%$ \\
\hline $3 \%$ & $3 \%$ \\
\hline $15 \%$ & $17 \%$ \\
\hline $28 \%$ & $27 \%$ \\
\hline $5 \%$ & $3 \%$ \\
\hline $35 \%$ & $31 \%$ \\
\hline $15 \%$ & $17 \%$ \\
\hline $98 \%$ & $100 \%$ \\
\hline $3 \%$ & $0 \%$ \\
\hline $3 \%$ & $0 \%$ \\
\hline $30 \%$ & $34 \%$ \\
\hline
\end{tabular}

$3 \%$

$3 \%$

$48 \%$

\begin{tabular}{|c|c|}
\hline $\begin{array}{l}\text { All participants at } \\
\text { baseline }(N=40)\end{array}$ & $\begin{array}{c}\text { Participants with } \\
\text { baseline and } 12- \\
\text { month data }(\mathbf{N}=29)\end{array}$ \\
\hline $48.2(10.7)$ & $48.0(10.0)$ \\
\hline $88 \%$ & $90 \%$ \\
\hline $30 \%$ & $28 \%$ \\
\hline $70 \%$ & $66 \%$ \\
\hline $3 \%$ & $3 \%$ \\
\hline $55 \%$ & $48 \%$ \\
\hline $18 \%$ & $24 \%$ \\
\hline $5 \%$ & $3 \%$ \\
\hline $18 \%$ & $17 \%$ \\
\hline $3 \%$ & $3 \%$ \\
\hline $3 \%$ & $3 \%$ \\
\hline $33 \%$ & $41 \%$ \\
\hline $33 \%$ & $31 \%$ \\
\hline $30 \%$ & $24 \%$ \\
\hline $3 \%$ & $3 \%$ \\
\hline $3 \%$ & $3 \%$ \\
\hline $0 \%$ & $0 \%$ \\
\hline $3 \%$ & $3 \%$ \\
\hline $15 \%$ & $17 \%$ \\
\hline $28 \%$ & $27 \%$ \\
\hline $5 \%$ & $3 \%$ \\
\hline $35 \%$ & $31 \%$ \\
\hline $15 \%$ & $17 \%$ \\
\hline $98 \%$ & $100 \%$ \\
\hline $3 \%$ & $0 \%$ \\
\hline $3 \%$ & $0 \%$ \\
\hline $30 \%$ & $34 \%$ \\
\hline
\end{tabular}

$24 \%$

\begin{tabular}{|c|c|}
\hline $\begin{array}{l}\text { All participants at } \\
\text { baseline }(N=40)\end{array}$ & $\begin{array}{c}\text { Participants with } \\
\text { baseline and } 12- \\
\text { month data }(\mathbf{N}=29)\end{array}$ \\
\hline $48.2(10.7)$ & $48.0(10.0)$ \\
\hline $88 \%$ & $90 \%$ \\
\hline $30 \%$ & $28 \%$ \\
\hline $70 \%$ & $66 \%$ \\
\hline $3 \%$ & $3 \%$ \\
\hline $55 \%$ & $48 \%$ \\
\hline $18 \%$ & $24 \%$ \\
\hline $5 \%$ & $3 \%$ \\
\hline $18 \%$ & $17 \%$ \\
\hline $3 \%$ & $3 \%$ \\
\hline $3 \%$ & $3 \%$ \\
\hline $33 \%$ & $41 \%$ \\
\hline $33 \%$ & $31 \%$ \\
\hline $30 \%$ & $24 \%$ \\
\hline $3 \%$ & $3 \%$ \\
\hline $3 \%$ & $3 \%$ \\
\hline $0 \%$ & $0 \%$ \\
\hline $3 \%$ & $3 \%$ \\
\hline $15 \%$ & $17 \%$ \\
\hline $28 \%$ & $27 \%$ \\
\hline $5 \%$ & $3 \%$ \\
\hline $35 \%$ & $31 \%$ \\
\hline $15 \%$ & $17 \%$ \\
\hline $98 \%$ & $100 \%$ \\
\hline $3 \%$ & $0 \%$ \\
\hline $3 \%$ & $0 \%$ \\
\hline $30 \%$ & $34 \%$ \\
\hline
\end{tabular}

\begin{tabular}{|c|c|}
\hline $\begin{array}{l}\text { All participants at } \\
\text { baseline }(N=40)\end{array}$ & $\begin{array}{c}\text { Participants with } \\
\text { baseline and } 12- \\
\text { month data }(\mathbf{N}=29)\end{array}$ \\
\hline $48.2(10.7)$ & $48.0(10.0)$ \\
\hline $88 \%$ & $90 \%$ \\
\hline $30 \%$ & $28 \%$ \\
\hline $70 \%$ & $66 \%$ \\
\hline $3 \%$ & $3 \%$ \\
\hline $55 \%$ & $48 \%$ \\
\hline $18 \%$ & $24 \%$ \\
\hline $5 \%$ & $3 \%$ \\
\hline $18 \%$ & $17 \%$ \\
\hline $3 \%$ & $3 \%$ \\
\hline $3 \%$ & $3 \%$ \\
\hline $33 \%$ & $41 \%$ \\
\hline $33 \%$ & $31 \%$ \\
\hline $30 \%$ & $24 \%$ \\
\hline $3 \%$ & $3 \%$ \\
\hline $3 \%$ & $3 \%$ \\
\hline $0 \%$ & $0 \%$ \\
\hline $3 \%$ & $3 \%$ \\
\hline $15 \%$ & $17 \%$ \\
\hline $28 \%$ & $27 \%$ \\
\hline $5 \%$ & $3 \%$ \\
\hline $35 \%$ & $31 \%$ \\
\hline $15 \%$ & $17 \%$ \\
\hline $98 \%$ & $100 \%$ \\
\hline $3 \%$ & $0 \%$ \\
\hline $3 \%$ & $0 \%$ \\
\hline $30 \%$ & $34 \%$ \\
\hline
\end{tabular}

$3 \%$

\begin{tabular}{|c|c|}
\hline $\begin{array}{l}\text { All participants at } \\
\text { baseline }(N=40)\end{array}$ & $\begin{array}{c}\text { Participants with } \\
\text { baseline and } 12- \\
\text { month data }(N=29)\end{array}$ \\
\hline $48.2(10.7)$ & $48.0(10.0)$ \\
\hline $88 \%$ & $90 \%$ \\
\hline $30 \%$ & $28 \%$ \\
\hline $70 \%$ & $66 \%$ \\
\hline $3 \%$ & $3 \%$ \\
\hline $55 \%$ & $48 \%$ \\
\hline $18 \%$ & $24 \%$ \\
\hline $5 \%$ & $3 \%$ \\
\hline $18 \%$ & $17 \%$ \\
\hline $3 \%$ & $3 \%$ \\
\hline $3 \%$ & $3 \%$ \\
\hline $33 \%$ & $41 \%$ \\
\hline $33 \%$ & $31 \%$ \\
\hline $30 \%$ & $24 \%$ \\
\hline $3 \%$ & $3 \%$ \\
\hline $3 \%$ & $3 \%$ \\
\hline $0 \%$ & $0 \%$ \\
\hline $3 \%$ & $3 \%$ \\
\hline $15 \%$ & $17 \%$ \\
\hline $28 \%$ & $27 \%$ \\
\hline $5 \%$ & $3 \%$ \\
\hline $35 \%$ & $31 \%$ \\
\hline $15 \%$ & $17 \%$ \\
\hline $98 \%$ & $100 \%$ \\
\hline $3 \%$ & $0 \%$ \\
\hline $3 \%$ & $0 \%$ \\
\hline $30 \%$ & $34 \%$ \\
\hline
\end{tabular}

\begin{tabular}{|c|c|}
\hline $\begin{array}{l}\text { All participants at } \\
\text { baseline }(N=40)\end{array}$ & $\begin{array}{c}\text { Participants with } \\
\text { baseline and } 12- \\
\text { month data }(N=29)\end{array}$ \\
\hline $48.2(10.7)$ & $48.0(10.0)$ \\
\hline $88 \%$ & $90 \%$ \\
\hline $30 \%$ & $28 \%$ \\
\hline $70 \%$ & $66 \%$ \\
\hline $3 \%$ & $3 \%$ \\
\hline $55 \%$ & $48 \%$ \\
\hline $18 \%$ & $24 \%$ \\
\hline $5 \%$ & $3 \%$ \\
\hline $18 \%$ & $17 \%$ \\
\hline $3 \%$ & $3 \%$ \\
\hline $3 \%$ & $3 \%$ \\
\hline $33 \%$ & $41 \%$ \\
\hline $33 \%$ & $31 \%$ \\
\hline $30 \%$ & $24 \%$ \\
\hline $3 \%$ & $3 \%$ \\
\hline $3 \%$ & $3 \%$ \\
\hline $0 \%$ & $0 \%$ \\
\hline $3 \%$ & $3 \%$ \\
\hline $15 \%$ & $17 \%$ \\
\hline $28 \%$ & $27 \%$ \\
\hline $5 \%$ & $3 \%$ \\
\hline $35 \%$ & $31 \%$ \\
\hline $15 \%$ & $17 \%$ \\
\hline $98 \%$ & $100 \%$ \\
\hline $3 \%$ & $0 \%$ \\
\hline $3 \%$ & $0 \%$ \\
\hline $30 \%$ & $34 \%$ \\
\hline
\end{tabular}

\begin{tabular}{|c|c|}
\hline $\begin{array}{l}\text { All participants at } \\
\text { baseline }(N=40)\end{array}$ & $\begin{array}{c}\text { Participants with } \\
\text { baseline and } 12- \\
\text { month data }(\mathbf{N}=29)\end{array}$ \\
\hline $48.2(10.7)$ & $48.0(10.0)$ \\
\hline $88 \%$ & $90 \%$ \\
\hline $30 \%$ & $28 \%$ \\
\hline $70 \%$ & $66 \%$ \\
\hline $3 \%$ & $3 \%$ \\
\hline $55 \%$ & $48 \%$ \\
\hline $18 \%$ & $24 \%$ \\
\hline $5 \%$ & $3 \%$ \\
\hline $18 \%$ & $17 \%$ \\
\hline $3 \%$ & $3 \%$ \\
\hline $3 \%$ & $3 \%$ \\
\hline $33 \%$ & $41 \%$ \\
\hline $33 \%$ & $31 \%$ \\
\hline $30 \%$ & $24 \%$ \\
\hline $3 \%$ & $3 \%$ \\
\hline $3 \%$ & $3 \%$ \\
\hline $0 \%$ & $0 \%$ \\
\hline $3 \%$ & $3 \%$ \\
\hline $15 \%$ & $17 \%$ \\
\hline $28 \%$ & $27 \%$ \\
\hline $5 \%$ & $3 \%$ \\
\hline $35 \%$ & $31 \%$ \\
\hline $15 \%$ & $17 \%$ \\
\hline $98 \%$ & $100 \%$ \\
\hline $3 \%$ & $0 \%$ \\
\hline $3 \%$ & $0 \%$ \\
\hline $30 \%$ & $34 \%$ \\
\hline
\end{tabular}

\begin{tabular}{|c|c|}
\hline $\begin{array}{l}\text { All participants at } \\
\text { baseline }(N=40)\end{array}$ & $\begin{array}{c}\text { Participants with } \\
\text { baseline and } 12- \\
\text { month data }(\mathbf{N}=29)\end{array}$ \\
\hline $48.2(10.7)$ & $48.0(10.0)$ \\
\hline $88 \%$ & $90 \%$ \\
\hline $30 \%$ & $28 \%$ \\
\hline $70 \%$ & $66 \%$ \\
\hline $3 \%$ & $3 \%$ \\
\hline $55 \%$ & $48 \%$ \\
\hline $18 \%$ & $24 \%$ \\
\hline $5 \%$ & $3 \%$ \\
\hline $18 \%$ & $17 \%$ \\
\hline $3 \%$ & $3 \%$ \\
\hline $3 \%$ & $3 \%$ \\
\hline $33 \%$ & $41 \%$ \\
\hline $33 \%$ & $31 \%$ \\
\hline $30 \%$ & $24 \%$ \\
\hline $3 \%$ & $3 \%$ \\
\hline $3 \%$ & $3 \%$ \\
\hline $0 \%$ & $0 \%$ \\
\hline $3 \%$ & $3 \%$ \\
\hline $15 \%$ & $17 \%$ \\
\hline $28 \%$ & $27 \%$ \\
\hline $5 \%$ & $3 \%$ \\
\hline $35 \%$ & $31 \%$ \\
\hline $15 \%$ & $17 \%$ \\
\hline $98 \%$ & $100 \%$ \\
\hline $3 \%$ & $0 \%$ \\
\hline $3 \%$ & $0 \%$ \\
\hline $30 \%$ & $34 \%$ \\
\hline
\end{tabular}

\begin{tabular}{|c|c|}
\hline $\begin{array}{l}\text { All participants at } \\
\text { baseline }(N=40)\end{array}$ & $\begin{array}{c}\text { Participants with } \\
\text { baseline and } 12- \\
\text { month data }(\mathbf{N}=29)\end{array}$ \\
\hline $48.2(10.7)$ & $48.0(10.0)$ \\
\hline $88 \%$ & $90 \%$ \\
\hline $30 \%$ & $28 \%$ \\
\hline $70 \%$ & $66 \%$ \\
\hline $3 \%$ & $3 \%$ \\
\hline $55 \%$ & $48 \%$ \\
\hline $18 \%$ & $24 \%$ \\
\hline $5 \%$ & $3 \%$ \\
\hline $18 \%$ & $17 \%$ \\
\hline $3 \%$ & $3 \%$ \\
\hline $3 \%$ & $3 \%$ \\
\hline $33 \%$ & $41 \%$ \\
\hline $33 \%$ & $31 \%$ \\
\hline $30 \%$ & $24 \%$ \\
\hline $3 \%$ & $3 \%$ \\
\hline $3 \%$ & $3 \%$ \\
\hline $0 \%$ & $0 \%$ \\
\hline $3 \%$ & $3 \%$ \\
\hline $15 \%$ & $17 \%$ \\
\hline $28 \%$ & $27 \%$ \\
\hline $5 \%$ & $3 \%$ \\
\hline $35 \%$ & $31 \%$ \\
\hline $15 \%$ & $17 \%$ \\
\hline $98 \%$ & $100 \%$ \\
\hline $3 \%$ & $0 \%$ \\
\hline $3 \%$ & $0 \%$ \\
\hline $30 \%$ & $34 \%$ \\
\hline
\end{tabular}

\begin{tabular}{|c|c|}
\hline $\begin{array}{l}\text { All participants at } \\
\text { baseline }(N=40)\end{array}$ & $\begin{array}{c}\text { Participants with } \\
\text { baseline and } 12- \\
\text { month data }(\mathbf{N}=29)\end{array}$ \\
\hline $48.2(10.7)$ & $48.0(10.0)$ \\
\hline $88 \%$ & $90 \%$ \\
\hline $30 \%$ & $28 \%$ \\
\hline $70 \%$ & $66 \%$ \\
\hline $3 \%$ & $3 \%$ \\
\hline $55 \%$ & $48 \%$ \\
\hline $18 \%$ & $24 \%$ \\
\hline $5 \%$ & $3 \%$ \\
\hline $18 \%$ & $17 \%$ \\
\hline $3 \%$ & $3 \%$ \\
\hline $3 \%$ & $3 \%$ \\
\hline $33 \%$ & $41 \%$ \\
\hline $33 \%$ & $31 \%$ \\
\hline $30 \%$ & $24 \%$ \\
\hline $3 \%$ & $3 \%$ \\
\hline $3 \%$ & $3 \%$ \\
\hline $0 \%$ & $0 \%$ \\
\hline $3 \%$ & $3 \%$ \\
\hline $15 \%$ & $17 \%$ \\
\hline $28 \%$ & $27 \%$ \\
\hline $5 \%$ & $3 \%$ \\
\hline $35 \%$ & $31 \%$ \\
\hline $15 \%$ & $17 \%$ \\
\hline $98 \%$ & $100 \%$ \\
\hline $3 \%$ & $0 \%$ \\
\hline $3 \%$ & $0 \%$ \\
\hline $30 \%$ & $34 \%$ \\
\hline
\end{tabular}

\begin{tabular}{|c|c|}
\hline $\begin{array}{l}\text { All participants at } \\
\text { baseline }(N=40)\end{array}$ & $\begin{array}{c}\text { Participants with } \\
\text { baseline and } 12- \\
\text { month data }(\mathbf{N}=29)\end{array}$ \\
\hline $48.2(10.7)$ & $48.0(10.0)$ \\
\hline $88 \%$ & $90 \%$ \\
\hline $30 \%$ & $28 \%$ \\
\hline $70 \%$ & $66 \%$ \\
\hline $3 \%$ & $3 \%$ \\
\hline $55 \%$ & $48 \%$ \\
\hline $18 \%$ & $24 \%$ \\
\hline $5 \%$ & $3 \%$ \\
\hline $18 \%$ & $17 \%$ \\
\hline $3 \%$ & $3 \%$ \\
\hline $3 \%$ & $3 \%$ \\
\hline $33 \%$ & $41 \%$ \\
\hline $33 \%$ & $31 \%$ \\
\hline $30 \%$ & $24 \%$ \\
\hline $3 \%$ & $3 \%$ \\
\hline $3 \%$ & $3 \%$ \\
\hline $0 \%$ & $0 \%$ \\
\hline $3 \%$ & $3 \%$ \\
\hline $15 \%$ & $17 \%$ \\
\hline $28 \%$ & $27 \%$ \\
\hline $5 \%$ & $3 \%$ \\
\hline $35 \%$ & $31 \%$ \\
\hline $15 \%$ & $17 \%$ \\
\hline $98 \%$ & $100 \%$ \\
\hline $3 \%$ & $0 \%$ \\
\hline $3 \%$ & $0 \%$ \\
\hline $30 \%$ & $34 \%$ \\
\hline
\end{tabular}

$3 \%$

$24 \%$

\begin{tabular}{|c|c|}
\hline $\begin{array}{l}\text { All participants at } \\
\text { baseline }(N=40)\end{array}$ & $\begin{array}{c}\text { Participants with } \\
\text { baseline and } 12- \\
\text { month data }(N=29)\end{array}$ \\
\hline $48.2(10.7)$ & $48.0(10.0)$ \\
\hline $88 \%$ & $90 \%$ \\
\hline $30 \%$ & $28 \%$ \\
\hline $70 \%$ & $66 \%$ \\
\hline $3 \%$ & $3 \%$ \\
\hline $55 \%$ & $48 \%$ \\
\hline $18 \%$ & $24 \%$ \\
\hline $5 \%$ & $3 \%$ \\
\hline $18 \%$ & $17 \%$ \\
\hline $3 \%$ & $3 \%$ \\
\hline $3 \%$ & $3 \%$ \\
\hline $33 \%$ & $41 \%$ \\
\hline $33 \%$ & $31 \%$ \\
\hline $30 \%$ & $24 \%$ \\
\hline $3 \%$ & $3 \%$ \\
\hline $3 \%$ & $3 \%$ \\
\hline $0 \%$ & $0 \%$ \\
\hline $3 \%$ & $3 \%$ \\
\hline $15 \%$ & $17 \%$ \\
\hline $28 \%$ & $27 \%$ \\
\hline $5 \%$ & $3 \%$ \\
\hline $35 \%$ & $31 \%$ \\
\hline $15 \%$ & $17 \%$ \\
\hline $98 \%$ & $100 \%$ \\
\hline $3 \%$ & $0 \%$ \\
\hline $3 \%$ & $0 \%$ \\
\hline $30 \%$ & $34 \%$ \\
\hline
\end{tabular}

\begin{tabular}{|c|c|}
\hline $\begin{array}{l}\text { All participants at } \\
\text { baseline }(N=40)\end{array}$ & $\begin{array}{c}\text { Participants with } \\
\text { baseline and } 12- \\
\text { month data }(\mathbf{N}=29)\end{array}$ \\
\hline $48.2(10.7)$ & $48.0(10.0)$ \\
\hline $88 \%$ & $90 \%$ \\
\hline $30 \%$ & $28 \%$ \\
\hline $70 \%$ & $66 \%$ \\
\hline $3 \%$ & $3 \%$ \\
\hline $55 \%$ & $48 \%$ \\
\hline $18 \%$ & $24 \%$ \\
\hline $5 \%$ & $3 \%$ \\
\hline $18 \%$ & $17 \%$ \\
\hline $3 \%$ & $3 \%$ \\
\hline $3 \%$ & $3 \%$ \\
\hline $33 \%$ & $41 \%$ \\
\hline $33 \%$ & $31 \%$ \\
\hline $30 \%$ & $24 \%$ \\
\hline $3 \%$ & $3 \%$ \\
\hline $3 \%$ & $3 \%$ \\
\hline $0 \%$ & $0 \%$ \\
\hline $3 \%$ & $3 \%$ \\
\hline $15 \%$ & $17 \%$ \\
\hline $28 \%$ & $27 \%$ \\
\hline $5 \%$ & $3 \%$ \\
\hline $35 \%$ & $31 \%$ \\
\hline $15 \%$ & $17 \%$ \\
\hline $98 \%$ & $100 \%$ \\
\hline $3 \%$ & $0 \%$ \\
\hline $3 \%$ & $0 \%$ \\
\hline $30 \%$ & $34 \%$ \\
\hline
\end{tabular}

\begin{tabular}{|c|c|}
\hline $\begin{array}{l}\text { All participants at } \\
\text { baseline }(N=40)\end{array}$ & $\begin{array}{c}\text { Participants with } \\
\text { baseline and } 12- \\
\text { month data }(\mathbf{N}=29)\end{array}$ \\
\hline $48.2(10.7)$ & $48.0(10.0)$ \\
\hline $88 \%$ & $90 \%$ \\
\hline $30 \%$ & $28 \%$ \\
\hline $70 \%$ & $66 \%$ \\
\hline $3 \%$ & $3 \%$ \\
\hline $55 \%$ & $48 \%$ \\
\hline $18 \%$ & $24 \%$ \\
\hline $5 \%$ & $3 \%$ \\
\hline $18 \%$ & $17 \%$ \\
\hline $3 \%$ & $3 \%$ \\
\hline $3 \%$ & $3 \%$ \\
\hline $33 \%$ & $41 \%$ \\
\hline $33 \%$ & $31 \%$ \\
\hline $30 \%$ & $24 \%$ \\
\hline $3 \%$ & $3 \%$ \\
\hline $3 \%$ & $3 \%$ \\
\hline $0 \%$ & $0 \%$ \\
\hline $3 \%$ & $3 \%$ \\
\hline $15 \%$ & $17 \%$ \\
\hline $28 \%$ & $27 \%$ \\
\hline $5 \%$ & $3 \%$ \\
\hline $35 \%$ & $31 \%$ \\
\hline $15 \%$ & $17 \%$ \\
\hline $98 \%$ & $100 \%$ \\
\hline $3 \%$ & $0 \%$ \\
\hline $3 \%$ & $0 \%$ \\
\hline $30 \%$ & $34 \%$ \\
\hline
\end{tabular}

\begin{tabular}{|c|c|}
\hline $\begin{array}{l}\text { All participants at } \\
\text { baseline }(N=40)\end{array}$ & $\begin{array}{c}\text { Participants with } \\
\text { baseline and } 12- \\
\text { month data }(N=29)\end{array}$ \\
\hline $48.2(10.7)$ & $48.0(10.0)$ \\
\hline $88 \%$ & $90 \%$ \\
\hline $30 \%$ & $28 \%$ \\
\hline $70 \%$ & $66 \%$ \\
\hline $3 \%$ & $3 \%$ \\
\hline $55 \%$ & $48 \%$ \\
\hline $18 \%$ & $24 \%$ \\
\hline $5 \%$ & $3 \%$ \\
\hline $18 \%$ & $17 \%$ \\
\hline $3 \%$ & $3 \%$ \\
\hline $3 \%$ & $3 \%$ \\
\hline $33 \%$ & $41 \%$ \\
\hline $33 \%$ & $31 \%$ \\
\hline $30 \%$ & $24 \%$ \\
\hline $3 \%$ & $3 \%$ \\
\hline $3 \%$ & $3 \%$ \\
\hline $0 \%$ & $0 \%$ \\
\hline $3 \%$ & $3 \%$ \\
\hline $15 \%$ & $17 \%$ \\
\hline $28 \%$ & $27 \%$ \\
\hline $5 \%$ & $3 \%$ \\
\hline $35 \%$ & $31 \%$ \\
\hline $15 \%$ & $17 \%$ \\
\hline $98 \%$ & $100 \%$ \\
\hline $3 \%$ & $0 \%$ \\
\hline $3 \%$ & $0 \%$ \\
\hline $30 \%$ & $34 \%$ \\
\hline
\end{tabular}

$28 \%$

\begin{tabular}{|c|c|}
\hline $\begin{array}{l}\text { All participants at } \\
\text { baseline }(N=40)\end{array}$ & $\begin{array}{c}\text { Participants with } \\
\text { baseline and } 12- \\
\text { month data }(\mathbf{N}=29)\end{array}$ \\
\hline $48.2(10.7)$ & $48.0(10.0)$ \\
\hline $88 \%$ & $90 \%$ \\
\hline $30 \%$ & $28 \%$ \\
\hline $70 \%$ & $66 \%$ \\
\hline $3 \%$ & $3 \%$ \\
\hline $55 \%$ & $48 \%$ \\
\hline $18 \%$ & $24 \%$ \\
\hline $5 \%$ & $3 \%$ \\
\hline $18 \%$ & $17 \%$ \\
\hline $3 \%$ & $3 \%$ \\
\hline $3 \%$ & $3 \%$ \\
\hline $33 \%$ & $41 \%$ \\
\hline $33 \%$ & $31 \%$ \\
\hline $30 \%$ & $24 \%$ \\
\hline $3 \%$ & $3 \%$ \\
\hline $3 \%$ & $3 \%$ \\
\hline $0 \%$ & $0 \%$ \\
\hline $3 \%$ & $3 \%$ \\
\hline $15 \%$ & $17 \%$ \\
\hline $28 \%$ & $27 \%$ \\
\hline $5 \%$ & $3 \%$ \\
\hline $35 \%$ & $31 \%$ \\
\hline $15 \%$ & $17 \%$ \\
\hline $98 \%$ & $100 \%$ \\
\hline $3 \%$ & $0 \%$ \\
\hline $3 \%$ & $0 \%$ \\
\hline $30 \%$ & $34 \%$ \\
\hline
\end{tabular}

\begin{tabular}{|c|c|}
\hline $\begin{array}{l}\text { All participants at } \\
\text { baseline }(N=40)\end{array}$ & $\begin{array}{c}\text { Participants with } \\
\text { baseline and } 12- \\
\text { month data }(\mathbf{N}=29)\end{array}$ \\
\hline $48.2(10.7)$ & $48.0(10.0)$ \\
\hline $88 \%$ & $90 \%$ \\
\hline $30 \%$ & $28 \%$ \\
\hline $70 \%$ & $66 \%$ \\
\hline $3 \%$ & $3 \%$ \\
\hline $55 \%$ & $48 \%$ \\
\hline $18 \%$ & $24 \%$ \\
\hline $5 \%$ & $3 \%$ \\
\hline $18 \%$ & $17 \%$ \\
\hline $3 \%$ & $3 \%$ \\
\hline $3 \%$ & $3 \%$ \\
\hline $33 \%$ & $41 \%$ \\
\hline $33 \%$ & $31 \%$ \\
\hline $30 \%$ & $24 \%$ \\
\hline $3 \%$ & $3 \%$ \\
\hline $3 \%$ & $3 \%$ \\
\hline $0 \%$ & $0 \%$ \\
\hline $3 \%$ & $3 \%$ \\
\hline $15 \%$ & $17 \%$ \\
\hline $28 \%$ & $27 \%$ \\
\hline $5 \%$ & $3 \%$ \\
\hline $35 \%$ & $31 \%$ \\
\hline $15 \%$ & $17 \%$ \\
\hline $98 \%$ & $100 \%$ \\
\hline $3 \%$ & $0 \%$ \\
\hline $3 \%$ & $0 \%$ \\
\hline $30 \%$ & $34 \%$ \\
\hline
\end{tabular}

\begin{tabular}{|c|c|}
\hline $\begin{array}{l}\text { All participants at } \\
\text { baseline }(N=40)\end{array}$ & $\begin{array}{c}\text { Participants with } \\
\text { baseline and } 12- \\
\text { month data }(\mathbf{N}=29)\end{array}$ \\
\hline $48.2(10.7)$ & $48.0(10.0)$ \\
\hline $88 \%$ & $90 \%$ \\
\hline $30 \%$ & $28 \%$ \\
\hline $70 \%$ & $66 \%$ \\
\hline $3 \%$ & $3 \%$ \\
\hline $55 \%$ & $48 \%$ \\
\hline $18 \%$ & $24 \%$ \\
\hline $5 \%$ & $3 \%$ \\
\hline $18 \%$ & $17 \%$ \\
\hline $3 \%$ & $3 \%$ \\
\hline $3 \%$ & $3 \%$ \\
\hline $33 \%$ & $41 \%$ \\
\hline $33 \%$ & $31 \%$ \\
\hline $30 \%$ & $24 \%$ \\
\hline $3 \%$ & $3 \%$ \\
\hline $3 \%$ & $3 \%$ \\
\hline $0 \%$ & $0 \%$ \\
\hline $3 \%$ & $3 \%$ \\
\hline $15 \%$ & $17 \%$ \\
\hline $28 \%$ & $27 \%$ \\
\hline $5 \%$ & $3 \%$ \\
\hline $35 \%$ & $31 \%$ \\
\hline $15 \%$ & $17 \%$ \\
\hline $98 \%$ & $100 \%$ \\
\hline $3 \%$ & $0 \%$ \\
\hline $3 \%$ & $0 \%$ \\
\hline $30 \%$ & $34 \%$ \\
\hline
\end{tabular}

\begin{tabular}{|c|c|}
\hline $\begin{array}{l}\text { All participants at } \\
\text { baseline }(N=40)\end{array}$ & $\begin{array}{c}\text { Participants with } \\
\text { baseline and } 12- \\
\text { month data }(\mathbf{N}=29)\end{array}$ \\
\hline $48.2(10.7)$ & $48.0(10.0)$ \\
\hline $88 \%$ & $90 \%$ \\
\hline $30 \%$ & $28 \%$ \\
\hline $70 \%$ & $66 \%$ \\
\hline $3 \%$ & $3 \%$ \\
\hline $55 \%$ & $48 \%$ \\
\hline $18 \%$ & $24 \%$ \\
\hline $5 \%$ & $3 \%$ \\
\hline $18 \%$ & $17 \%$ \\
\hline $3 \%$ & $3 \%$ \\
\hline $3 \%$ & $3 \%$ \\
\hline $33 \%$ & $41 \%$ \\
\hline $33 \%$ & $31 \%$ \\
\hline $30 \%$ & $24 \%$ \\
\hline $3 \%$ & $3 \%$ \\
\hline $3 \%$ & $3 \%$ \\
\hline $0 \%$ & $0 \%$ \\
\hline $3 \%$ & $3 \%$ \\
\hline $15 \%$ & $17 \%$ \\
\hline $28 \%$ & $27 \%$ \\
\hline $5 \%$ & $3 \%$ \\
\hline $35 \%$ & $31 \%$ \\
\hline $15 \%$ & $17 \%$ \\
\hline $98 \%$ & $100 \%$ \\
\hline $3 \%$ & $0 \%$ \\
\hline $3 \%$ & $0 \%$ \\
\hline $30 \%$ & $34 \%$ \\
\hline
\end{tabular}

\begin{tabular}{|c|c|}
\hline $\begin{array}{l}\text { All participants at } \\
\text { baseline }(N=40)\end{array}$ & $\begin{array}{c}\text { Participants with } \\
\text { baseline and } 12- \\
\text { month data }(N=29)\end{array}$ \\
\hline $48.2(10.7)$ & $48.0(10.0)$ \\
\hline $88 \%$ & $90 \%$ \\
\hline $30 \%$ & $28 \%$ \\
\hline $70 \%$ & $66 \%$ \\
\hline $3 \%$ & $3 \%$ \\
\hline $55 \%$ & $48 \%$ \\
\hline $18 \%$ & $24 \%$ \\
\hline $5 \%$ & $3 \%$ \\
\hline $18 \%$ & $17 \%$ \\
\hline $3 \%$ & $3 \%$ \\
\hline $3 \%$ & $3 \%$ \\
\hline $33 \%$ & $41 \%$ \\
\hline $33 \%$ & $31 \%$ \\
\hline $30 \%$ & $24 \%$ \\
\hline $3 \%$ & $3 \%$ \\
\hline $3 \%$ & $3 \%$ \\
\hline $0 \%$ & $0 \%$ \\
\hline $3 \%$ & $3 \%$ \\
\hline $15 \%$ & $17 \%$ \\
\hline $28 \%$ & $27 \%$ \\
\hline $5 \%$ & $3 \%$ \\
\hline $35 \%$ & $31 \%$ \\
\hline $15 \%$ & $17 \%$ \\
\hline $98 \%$ & $100 \%$ \\
\hline $3 \%$ & $0 \%$ \\
\hline $3 \%$ & $0 \%$ \\
\hline $30 \%$ & $34 \%$ \\
\hline
\end{tabular}

\begin{tabular}{|c|c|}
\hline $\begin{array}{l}\text { All participants at } \\
\text { baseline }(N=40)\end{array}$ & $\begin{array}{c}\text { Participants with } \\
\text { baseline and } 12- \\
\text { month data }(\mathbf{N}=29)\end{array}$ \\
\hline $48.2(10.7)$ & $48.0(10.0)$ \\
\hline $88 \%$ & $90 \%$ \\
\hline $30 \%$ & $28 \%$ \\
\hline $70 \%$ & $66 \%$ \\
\hline $3 \%$ & $3 \%$ \\
\hline $55 \%$ & $48 \%$ \\
\hline $18 \%$ & $24 \%$ \\
\hline $5 \%$ & $3 \%$ \\
\hline $18 \%$ & $17 \%$ \\
\hline $3 \%$ & $3 \%$ \\
\hline $3 \%$ & $3 \%$ \\
\hline $33 \%$ & $41 \%$ \\
\hline $33 \%$ & $31 \%$ \\
\hline $30 \%$ & $24 \%$ \\
\hline $3 \%$ & $3 \%$ \\
\hline $3 \%$ & $3 \%$ \\
\hline $0 \%$ & $0 \%$ \\
\hline $3 \%$ & $3 \%$ \\
\hline $15 \%$ & $17 \%$ \\
\hline $28 \%$ & $27 \%$ \\
\hline $5 \%$ & $3 \%$ \\
\hline $35 \%$ & $31 \%$ \\
\hline $15 \%$ & $17 \%$ \\
\hline $98 \%$ & $100 \%$ \\
\hline $3 \%$ & $0 \%$ \\
\hline $3 \%$ & $0 \%$ \\
\hline $30 \%$ & $34 \%$ \\
\hline
\end{tabular}

\begin{tabular}{|c|c|}
\hline $\begin{array}{l}\text { All participants at } \\
\text { baseline }(N=40)\end{array}$ & $\begin{array}{c}\text { Participants with } \\
\text { baseline and } 12- \\
\text { month data }(\mathbf{N}=29)\end{array}$ \\
\hline $48.2(10.7)$ & $48.0(10.0)$ \\
\hline $88 \%$ & $90 \%$ \\
\hline $30 \%$ & $28 \%$ \\
\hline $70 \%$ & $66 \%$ \\
\hline $3 \%$ & $3 \%$ \\
\hline $55 \%$ & $48 \%$ \\
\hline $18 \%$ & $24 \%$ \\
\hline $5 \%$ & $3 \%$ \\
\hline $18 \%$ & $17 \%$ \\
\hline $3 \%$ & $3 \%$ \\
\hline $3 \%$ & $3 \%$ \\
\hline $33 \%$ & $41 \%$ \\
\hline $33 \%$ & $31 \%$ \\
\hline $30 \%$ & $24 \%$ \\
\hline $3 \%$ & $3 \%$ \\
\hline $3 \%$ & $3 \%$ \\
\hline $0 \%$ & $0 \%$ \\
\hline $3 \%$ & $3 \%$ \\
\hline $15 \%$ & $17 \%$ \\
\hline $28 \%$ & $27 \%$ \\
\hline $5 \%$ & $3 \%$ \\
\hline $35 \%$ & $31 \%$ \\
\hline $15 \%$ & $17 \%$ \\
\hline $98 \%$ & $100 \%$ \\
\hline $3 \%$ & $0 \%$ \\
\hline $3 \%$ & $0 \%$ \\
\hline $30 \%$ & $34 \%$ \\
\hline
\end{tabular}

$3 \%$

\begin{tabular}{|c|c|}
\hline $\begin{array}{l}\text { All participants at } \\
\text { baseline }(N=40)\end{array}$ & $\begin{array}{c}\text { Participants with } \\
\text { baseline and } 12- \\
\text { month data }(\mathbf{N}=29)\end{array}$ \\
\hline $48.2(10.7)$ & $48.0(10.0)$ \\
\hline $88 \%$ & $90 \%$ \\
\hline $30 \%$ & $28 \%$ \\
\hline $70 \%$ & $66 \%$ \\
\hline $3 \%$ & $3 \%$ \\
\hline $55 \%$ & $48 \%$ \\
\hline $18 \%$ & $24 \%$ \\
\hline $5 \%$ & $3 \%$ \\
\hline $18 \%$ & $17 \%$ \\
\hline $3 \%$ & $3 \%$ \\
\hline $3 \%$ & $3 \%$ \\
\hline $33 \%$ & $41 \%$ \\
\hline $33 \%$ & $31 \%$ \\
\hline $30 \%$ & $24 \%$ \\
\hline $3 \%$ & $3 \%$ \\
\hline $3 \%$ & $3 \%$ \\
\hline $0 \%$ & $0 \%$ \\
\hline $3 \%$ & $3 \%$ \\
\hline $15 \%$ & $17 \%$ \\
\hline $28 \%$ & $27 \%$ \\
\hline $5 \%$ & $3 \%$ \\
\hline $35 \%$ & $31 \%$ \\
\hline $15 \%$ & $17 \%$ \\
\hline $98 \%$ & $100 \%$ \\
\hline $3 \%$ & $0 \%$ \\
\hline $3 \%$ & $0 \%$ \\
\hline $30 \%$ & $34 \%$ \\
\hline
\end{tabular}

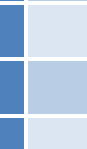

\begin{tabular}{|c|c|}
\hline $3 \%$ & $0 \%$ \\
\hline $30 \%$ & $34 \%$ \\
\hline $65 \%$ & $66 \%$ \\
\hline & \\
\hline $0 \%$ & $0 \%$ \\
\hline $10 \%$ & $21 \%$ \\
\hline $90 \%$ & $79 \%$ \\
\hline $10 \%$ & $41 \%$ \\
\hline $15 \%$ & $21 \%$ \\
\hline
\end{tabular}


reporting higher rates of diabetes at baseline $(P=0.012)$. No other differences were observed between the two groups.

Fig 1. Flowchart of participants

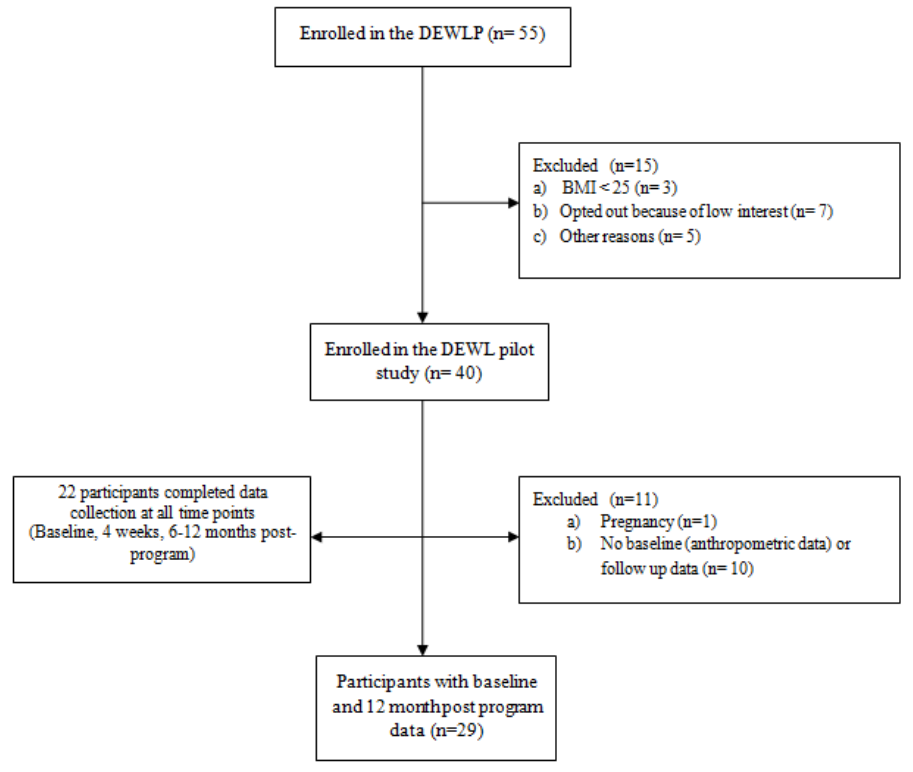

Impact of Intervention

At baseline, participants enrolled in the study weighed an average of $97.8 \mathrm{~kg}(S D \pm 17.05)$ (Table II). Of participants $(\mathrm{N}=22)$ with weight data at all times points (baseline, 4 weeks, 6 and 12 months post-program), the mean weight was $98.7 \mathrm{~kg}$ (SD \pm $18.1), 95.7 \mathrm{~kg}(\mathrm{SD} \pm 17.6), 94.3 \mathrm{~kg}$ (SD \pm 16.2 )and $96.4 \mathrm{~kg}$ (SD \pm 16.9), at baseline, 4 weeks, and 6 and 12 months post-program, respectively (Fig 2 ).

Fig 2. Weight change in kilograms by time point

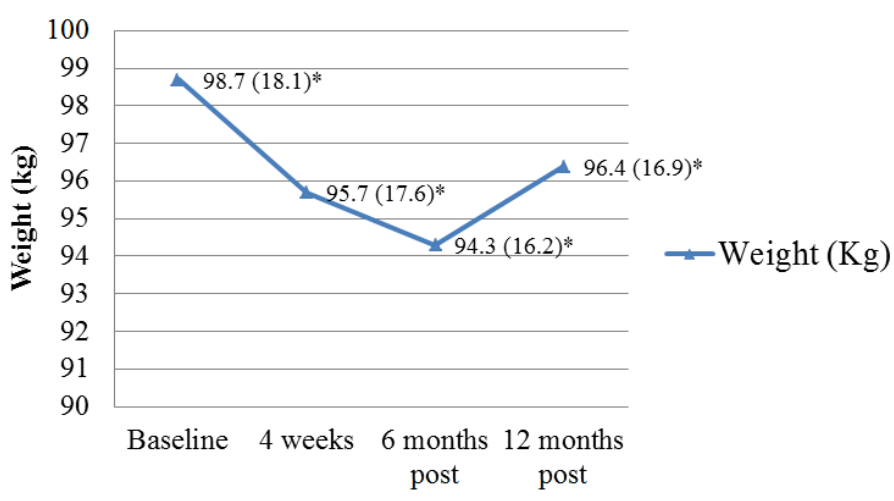

*weight (SD). $\mathrm{N}$ includes only participants with data at all time points.

Participants who completed both baseline and 12 months post program data collection ( $\mathrm{N}=29)$ lost an average of $2.7 \mathrm{~kg}(2.8 \%)$ $(S D \pm 6.35)(p=0.03)$. One-way repeated measures ANOVA indicated a significant effect of time on weight and BMI $(p<0.001$ and $p<0.001)$. Post-hoc tests revealed a weight reduction from baseline to 4 weeks, baseline to 6 months post -program and baseline to 12 months post -program ( $p<0.000, p<0.001, p=$ 0.03 ), respectively. Post hoc paired samples t-tests revealed no significant differences in weight from 4 weeks to 6 and 12 months post-program ( $p=0.26 ; p=0.98$ ), respectively. A post hoc paired samples t-test revealed a significant increase in weight from 6 month post program to 12 months post -program
Table II Participant weight, BMI, percent body fat and blood pressure change by time point

\begin{tabular}{|c|c|c|c|c|c|}
\hline Interval & $\begin{array}{l}\text { Weight } \\
\text { M (SD) }\end{array}$ & $\begin{array}{c}\text { BMI } \\
\text { M (SD) }\end{array}$ & $\begin{array}{c}\% \text { Body } \\
\text { Fat } \\
\text { M (SD) }\end{array}$ & $\begin{array}{c}\text { SBP } \\
\text { M (SD) }\end{array}$ & $\begin{array}{c}\text { DBP } \\
\text { M (SD) }\end{array}$ \\
\hline & $\mathrm{N}=38$ & $\mathrm{~N}=38$ & $\mathrm{~N}=38$ & $\mathrm{~N}=37$ & $\mathrm{~N}=37$ \\
\hline Baseline & $\begin{array}{c}98.7 \\
(17.02)\end{array}$ & $\begin{array}{c}37.3 \\
(6.69)\end{array}$ & $\begin{array}{c}45.4 \\
(6.30)\end{array}$ & $\begin{array}{c}118.8 \\
(15.16)\end{array}$ & $\begin{array}{c}77.7 \\
(9.59)\end{array}$ \\
\hline \multirow[t]{2}{*}{$\begin{array}{l}4 \text { weeks } \\
\text { Post } \\
\text { program }\end{array}$} & $\begin{array}{c}96.0 \\
(16.59)\end{array}$ & $\begin{array}{c}36.3 \\
(6.63)\end{array}$ & $\begin{array}{c}43.2 \\
(6.55)\end{array}$ & $\begin{array}{c}119.3 \\
(15.25)\end{array}$ & $\begin{array}{c}76.5 \\
(8.95)\end{array}$ \\
\hline & $\mathrm{N}=27$ & $\mathrm{~N}=27$ & $\mathrm{~N}=26$ & $\mathrm{~N}=27$ & $\mathrm{~N}=27$ \\
\hline Baseline & $\begin{array}{c}97.9 \\
(16.96)\end{array}$ & $\begin{array}{c}37.0 \\
(6.94)\end{array}$ & $\begin{array}{c}45.6 \\
(6.12)\end{array}$ & $\begin{array}{c}117.1 \\
(15.23)\end{array}$ & $\begin{array}{c}76.9 \\
(10.68)\end{array}$ \\
\hline \multirow[t]{2}{*}{$\begin{array}{c}6 \text { months } \\
\text { post } \\
\text { program }\end{array}$} & $\begin{array}{c}93.5 \\
(15.36)\end{array}$ & $\begin{array}{c}35.4 \\
(6.65)\end{array}$ & $\begin{array}{c}41.6 \\
(7.36)\end{array}$ & $\begin{array}{c}120.4 \\
(18.83)\end{array}$ & $\begin{array}{c}77.9 \\
(10.71)\end{array}$ \\
\hline & $\mathrm{N}=29$ & $N=29$ & $\mathrm{~N}=29$ & $\mathrm{~N}=29$ & $\mathrm{~N}=29$ \\
\hline Baseline & $\begin{array}{c}98.9 \\
(17.35)\end{array}$ & $\begin{array}{c}37.8 \\
(7.28)\end{array}$ & $\begin{array}{c}46.1 \\
(6.30)\end{array}$ & $\begin{array}{c}118.5 \\
(14.86)\end{array}$ & $\begin{array}{c}77.3 \\
(10.28)\end{array}$ \\
\hline \multirow[t]{2}{*}{$\begin{array}{c}12 \\
\text { months } \\
\text { post } \\
\text { program }\end{array}$} & $\begin{array}{c}96.2 \\
(15.95)\end{array}$ & $\begin{array}{c}36.8 \\
(6.86)\end{array}$ & $\begin{array}{c}43.7 \\
(6.83)\end{array}$ & $\begin{array}{c}118.3 \\
(13.37)\end{array}$ & $\begin{array}{c}77.1 \\
(8.88)\end{array}$ \\
\hline & $\mathrm{N}=26$ & $N=26$ & $\mathrm{~N}=25$ & $\mathrm{~N}=25$ & $\mathrm{~N}=25$ \\
\hline $\begin{array}{c}4 \text { weeks } \\
\text { post } \\
\text { program }\end{array}$ & $\begin{array}{c}95.4 \\
(16.41)\end{array}$ & $\begin{array}{c}35.9 \\
(6.99)\end{array}$ & $\begin{array}{c}43.5 \\
(6.20)\end{array}$ & $\begin{array}{c}117.2 \\
(14.14)\end{array}$ & $\begin{array}{c}75.7 \\
(10.01)\end{array}$ \\
\hline \multirow[t]{2}{*}{$\begin{array}{c}6 \text { months } \\
\text { post } \\
\text { program }\end{array}$} & $\begin{array}{c}94.1 \\
(15.27)\end{array}$ & $\begin{array}{c}35.5 \\
(6.78)\end{array}$ & $\begin{array}{c}41.5 \\
(7.50)\end{array}$ & $\begin{array}{c}120.8 \\
(19.34)\end{array}$ & $\begin{array}{c}77.9 \\
(11.03)\end{array}$ \\
\hline & $\mathrm{N}=27$ & $N=27$ & $\mathrm{~N}=27$ & $N=26$ & $N=26$ \\
\hline $\begin{array}{l}4 \text { weeks } \\
\text { post } \\
\text { program }\end{array}$ & $\begin{array}{c}97.4 \\
(16.88)\end{array}$ & $\begin{array}{c}37.1 \\
(7.39)\end{array}$ & $\begin{array}{c}43.8 \\
(6.70)\end{array}$ & $\begin{array}{c}119.8 \\
(13.16)\end{array}$ & $\begin{array}{c}77.2 \\
(8.51)\end{array}$ \\
\hline \multirow[t]{2}{*}{$\begin{array}{c}12 \\
\text { months } \\
\text { post } \\
\text { program }\end{array}$} & $\begin{array}{c}97.3 \\
(15.91)\end{array}$ & $\begin{array}{c}37.1 \\
(7.03)\end{array}$ & $\begin{array}{c}44.0 \\
(6.99)\end{array}$ & $\begin{array}{c}119.6 \\
(13.49)\end{array}$ & $\begin{array}{c}78.1 \\
(8.78)\end{array}$ \\
\hline & $\mathrm{N}=23$ & $\mathrm{~N}=23$ & $\mathrm{~N}=23$ & $\mathrm{~N}=23$ & $\mathrm{~N}=23$ \\
\hline $\begin{array}{c}6 \text { months } \\
\text { post } \\
\text { program }\end{array}$ & $\begin{array}{c}93.5 \\
(16.32)\end{array}$ & $\begin{array}{c}35.9 \\
(7.09)\end{array}$ & $\begin{array}{c}41.8 \\
(7.70)\end{array}$ & $\begin{array}{c}122.0 \\
(18.63)\end{array}$ & $\begin{array}{c}78.7 \\
(10.52)\end{array}$ \\
\hline $\begin{array}{c}12 \\
\text { months } \\
\text { post } \\
\text { program }\end{array}$ & $\begin{array}{c}95.6 \\
(17.02)\end{array}$ & $\begin{array}{c}36.7 \\
(7.22)\end{array}$ & $\begin{array}{c}43.9 \\
(6.78)\end{array}$ & $\begin{array}{c}117.4 \\
(13.84)\end{array}$ & $\begin{array}{c}76.1 \\
(8.50)\end{array}$ \\
\hline
\end{tabular}

( $p$ =0.004). BMl followed a similar pattern than weight. However, BMI decreased was significant from 4 weeks to 6 and 12 months post program. Weight regained was significant from 4 weeks to 12 months post-program $(P=0.004)$ (Table III).

For our secondary outcomes, one-way repeated measures ANOVA indicated a significant change over time in percent body fat, a reduction of $2.4 \%$ (SD \pm 3.86 )from baseline to 12 months post -program $(p=0.002)$. Post hoc, paired samples t-tests revealed a significant decrease in percent body fat between 
an automated baseline to 4 weeks and 6 and 12 months post intervention $(p=0.000, p=0.001, p=0.002)$, respectively. Similar to weight no significant difference was observed from 4 weeks to 6 and 12 months follow up ( $p=0.12$ and $p=0.71)$, respectively.

No significant change over time was observed in blood pressure (systolic and diastolic).

\section{Discussion}

The DEWL program was designed to provide a concentrated intervention for weight change, delivered in a "mini-immersion" program to employees and their dependents. The purpose of this study was to set the stage for a large clinical trial by assessing whether we could feasibly evaluate the impact of the program over time, using rigorous methodology and participants who were paying to participate in the DEWL program itself. We also assessed preliminary effect size measures of the program's impact on weight, BMl, body fat percentage and blood pressure. In terms of feasibility, $73 \%$ of the participants paying for the DEWL program were willing to enroll in the measurement study, and $73 \%$ of those enrolled completed data collection at 12 month post intervention. Further, $55 \%$ of participants completed our rigorous data collection process at all-time points. In terms of program impact, participants had lost an average of $4.4 \mathrm{~kg}(9.7$ lbs) at 6 months post program and $2.7 \mathrm{~kg}(5.9 \mathrm{lbs})$ at 12 months post program. The finding of weight regain between 6 and 12 months suggests that a structured ongoing support activity or a program refresher between 6 and 12 months might have been helpful.

Our study retention rate was superior or similar to those previously reported. For example, Salinardi, et al testing a weight loss intervention in a Boston worksite reported retention at 6 months post weight loss interventions of $89 \%$. In our study, the retention at 6 months was $67.5 \%$. Further, only $42 \%$ of participants in that study completed the 12 months structure weight loss maintenance program [19]. Attrition rates in clinical trials of other obesity treatment programs have been reported to be between $30-50 \%$ [20]. Given both the $73 \%$ enrollment rate, and the $73 \%$ retention rate at 12 months follow-up, the process of studying outcomes on participants paying to undergo the DEWL worksite program does indeed seem feasible. One issue that will need to be addressed in a larger trial is the differential rate of drop-out for those with diabetes. Other weight loss studies with patients with diabetes testing lifestyle interventions for weight loss outside the worksite have reported a retention rate of $>95 \%$ at one year [21].

In terms of the estimated impact of this worksite program, our findings are consistent with other worksite studies using longer, but less intensive interventions. For example, one systematic review of worksite programs found that a large range of interventions (including those that were informational, those teaching behavioral skills, and those that targeted policy and environmental changes) all demonstrated a modest reduction in weight averaging $2.8 \mathrm{lbs}$ at 12 months[10]. Another study, by Lahiri et al published in 2012, demonstrated a $3.4 \mathrm{~kg}$ (7.5 lbs)
Table III Mean Differences of Primary and Secondary Outcomes for Participants who Completed the study

\begin{tabular}{|c|c|c|c|c|c|c|}
\hline & & $\begin{array}{c}\text { Mean } \\
\text { Difference }\end{array}$ & SD & $\mathrm{t}$ & df & $\mathrm{p}$ \\
\hline \multirow[t]{6}{*}{$\begin{array}{l}\text { Weight } \\
(\mathrm{kg})\end{array}$} & Baseline -4 weeks & -2.818 & 1.647 & 8.889 & 26 & 0.000 \\
\hline & $\begin{array}{l}\text { Baseline - } 6 \\
\text { months }\end{array}$ & -4.358 & 5.614 & 3.723 & 22 & 0.001 \\
\hline & $\begin{array}{l}\text { Baseline - } 12 \\
\text { months }\end{array}$ & -2.700 & 6.348 & 2.291 & 28 & 0.03 \\
\hline & $\begin{array}{l}4 \text { weeks - } 6 \\
\text { months }\end{array}$ & -1.338 & 5.432 & 1.156 & 21 & 0.26 \\
\hline & $\begin{array}{c}4 \text { weeks - } 12 \\
\text { months }\end{array}$ & -0.040 & 6.553 & 0.031 & 26 & 0.98 \\
\hline & $\begin{array}{l}6 \text { months - } 12 \\
\text { months }\end{array}$ & +2.065 & 3.104 & -3.191 & 22 & 0.004 \\
\hline \multirow{6}{*}{$\begin{array}{l}\text { Body } \\
\text { Mass } \\
\text { Index } \\
\text { (BMI) }\end{array}$} & Baseline -4 weeks & -1.056 & .598 & 9.172 & 26 & 0.000 \\
\hline & $\begin{array}{l}\text { Baseline - } 6 \\
\text { months }\end{array}$ & -1.615 & 1.904 & 4.068 & 22 & 0.001 \\
\hline & $\begin{array}{c}\text { Baseline - } 12 \\
\text { months }\end{array}$ & -1.019 & 2.439 & 2.249 & 28 & 0.000 \\
\hline & $\begin{array}{c}4 \text { weeks - } 6 \\
\text { months }\end{array}$ & -0.469 & 1.896 & 1.159 & 21 & 0.001 \\
\hline & $\begin{array}{c}4 \text { weeks - } 12 \\
\text { months }\end{array}$ & 0.018 & 2.547 & 0.036 & 26 & 0.03 \\
\hline & $\begin{array}{l}6 \text { months - } 12 \\
\text { months }\end{array}$ & +.774 & 1.154 & -3.217 & 22 & 0.26 \\
\hline \multirow[t]{12}{*}{$\begin{array}{l}\% \text { Body } \\
\text { Fat }\end{array}$} & Baseline - 4 weeks & -2.663 & 2.965 & 4.667 & 26 & 0.000 \\
\hline & $\begin{array}{l}\text { Baseline - } 6 \\
\text { months }\end{array}$ & -4.265 & 5.361 & 3.815 & 22 & 0.001 \\
\hline & $\begin{array}{l}\text { Baseline - } 12 \\
\text { months }\end{array}$ & -2.398 & 3.864 & 3.342 & 28 & 0.002 \\
\hline & $\begin{array}{l}4 \text { weeks - } 6 \\
\text { months }\end{array}$ & -1.977 & 5.691 & 1.630 & 21 & 0.12 \\
\hline & $\begin{array}{c}4 \text { weeks - } 12 \\
\text { months }\end{array}$ & +0.219 & 3.000 & -0.380 & 26 & 0.71 \\
\hline & $\begin{array}{c}6 \text { months - } 12 \\
\text { months }\end{array}$ & +2.173 & 5.353 & -1.947 & 22 & 0.064 \\
\hline & Baseline -4 weeks & -0.115 & 11.047 & 0.053 & 25 & 0.958 \\
\hline & $\begin{array}{l}\text { Baseline - } 6 \\
\text { months }\end{array}$ & +4.087 & 13.163 & -1.489 & 22 & 0.151 \\
\hline & $\begin{array}{l}\text { Baseline - } 12 \\
\text { months }\end{array}$ & -0.138 & 10.822 & 0.069 & 28 & 0.946 \\
\hline & $\begin{array}{l}4 \text { weeks - } 6 \\
\text { months }\end{array}$ & +4.238 & 16.343 & -1.188 & 20 & 0.249 \\
\hline & $\begin{array}{l}4 \text { weeks }-12 \\
\text { months }\end{array}$ & -0.192 & 12.490 & 0.079 & 25 & 0.938 \\
\hline & $\begin{array}{l}6 \text { months - } 12 \\
\text { months }\end{array}$ & -4.609 & 17.201 & 1.285 & 22 & 0.212 \\
\hline
\end{tabular}


weight loss over 28 weeks in a worksite group "incentivized" to participate in a weight loss intervention [22]. In our study, participants lost $4.4 \mathrm{~kg}$ (9.7 lbs) at 6 months post intervention and $2.7 \mathrm{Kg}(5.9 \mathrm{lbs})$ at 12 months post intervention indicating that our intervention is potentially more effective than other interventions both at 6 and 12 months post intervention. One can speculate that the larger weight loss seen in our study is in part due to studying participants who were highly motivated, given their willingness to pay for the DEWL program. Nonetheless, other studies of ongoing weight loss programs do not necessarily demonstrate such outcomes. For example, in a study conducted by Thorndike, et al (2011) employees of Massachusetts General Hospital (MGH) that participated in a structured 10-week wellness program to improve nutrition and exercise habits demonstrated an average weight loss of $0.4 \mathrm{~kg}(0.88 \mathrm{lbs})$ at one year follow-up[23]. A second possible explanation of the relative effectiveness of the DEWL approach may be the comprehensive nature of the program, with multiple weekly contacts, including group educational sessions, exercise classes and the provision of calorie-controlled meals. This explanation is consistent with the conclusion reported by multiple researchers [24-28] that more intense interventions have greater impact on participant outcomes. Interestingly, our participants continued to lose weight after completing the 4 week program, reaching their lowest weight 6 months post intervention. It is possible that participants with initial weight loss continued to be motivated and were thus able to sustain their new lifestyles longer after completing the program, although this remains an empirical question. By the end of the study, participants had regained some weight but weighted less than their entry weight, consistent with findings from other research. For example, participants of a weight loss maintenance study regained some weight over $21 / 2$ years, following an initial weight loss of at least $4 \mathrm{~kg}$. However, $71 \%$ of participants had a weight below their entry weight at the end of the trial [17]. Similarly, a systematic review of dietary counseling and weight loss showed that at 3 year follow up participants regain half of their initial weight loss [25]. Oxford Advanced Learners' Dictionary. Oxford University Press, New Delhi.7th edition 2005, Pg-1779.

Results from our study did not show a significant lowering of blood pressure. Despite the well-known blood pressure lowering effect of weight loss in the overweight/obese[16-18, 26], this finding is not surprising given that only $10 \%$ of our participants reported hypertension at baseline and baseline blood pressure was normal in most participants. In a larger trial, we will need to carefully consider inclusion criteria if we are to evaluate the potential impact of the DEWL program on blood pressure. We were, nonetheless, pleased that participants did not complain about the rigorous measurement procedures used in collecting this data.

\section{Limitations and Strengths}

Limitations of our study include the small sample size, lack of a control group, and limited external validity. Specifically, our sample represents a small subset of Duke Employees and dependents (Duke has a total of 35, 510 employees with a wide range of ages and salary), who were mostly middle-aged women, with relatively high educational and income levels. In addition, participants in our study had paid $\$ 650$ dollars and accepted a substantial time commitment to enroll in the DEWL program prior to being invited to join the research project. As such, our participants may represent a highly motivated group. Given the design of the study, it is difficult to assess the relative impact of motivation on the initial and subsequent weight loss. Another limitation is that we did not determine the extent to which participants actually engaged in the four week program, or what impact degree of engagement had on outcomes. Similarly, we are unable to assess which program elements - for example, educational classes, exercise classes, the provision of meals, etc. were most strongly related with successful outcomes. Finally, we do not know the importance of participation in the optional postprogram extension. We know which participants purchased extended time after the four weeks, but have no data on how many meals they consumed at the DFC or how many exercise class they attended. In a larger, subsequent trial, we will need to carefully track attendance and utilization of all components of the program, as well as to track the behaviors of participants during the follow-up time period. Despite these limitations, our findings do indicate that a larger trial of this brief but intensive intervention would be feasible. Three-quarters of the program participants were willing to participate in the measurement study, and almost three-quarters of those who participated in the study were retained at one-year follow-up. Moreover, these initial estimates of the impact of the DEWL program show that it is potentially effective in accomplishing medically meaningful weight loss over the course of four weeks, with maintenance of some of that weight loss 12 months after completing the intervention. Future research in a larger scale trial with a more diverse population is needed to more firmly establish effectiveness of this approach and determine strategies for cost-effective implementation and sustainability. Evaluation of the experience of greater numbers of participants will also be important in helping us to understand participant characteristics and program elements that may be associated with more successful outcomes.

\section{References}

1. Linnan L, Tate DF, Harrington CB, et al. Organizational- and employee-level recruitment into a worksite-based weight loss study. Clin Trials. 2012;9:215-225.

2. Thorndike AN. Workplace Interventions to Reduce Obesity and Cardiometabolic Risk. Curr Cardiovasc Risk Rep. 2011;5:79-85.

3. Ostbye T, Dement JM, Krause KM. Obesity and workers' compensation: results from the Duke Health and Safety Surveillance System. Archives of internal medicine. 2007; 167:766-773.

4. Finkelstein EA, DiBonaventura M, Burgess SM, Hale BC. The costs of obesity in the workplace. J Occup Environ Med. 2010;52:971-976.

5. Lemon SC, Pratt CA. Worksite environmental interventions for obesity control: an overview. J Occup Environ Med. 2010;52 Suppl 1:S1-3. 
6. French SA, Hannan PJ, Harnack LJ, Mitchell NR, Toomey TL, Gerlach A. Pricing and availability intervention in vending machines at four bus garages. J Occup Environ Med. 2010;52 Suppl 1:S29-33.

7. Nigg CR, Albright C, Williams R, et al. Are physical activity and nutrition indicators of the checklist of health promotion environments at worksites [CHEW] associated with employee obesity among hotel workers? J Occup Environ Med. 2010;52 Suppl 1:S4-7.

8. Petersen R, Sill S, Lu C, Young J, Edington DW. Effectiveness of employee internet-based weight management program. J Occup Environ Med. 2008;50:163171.

9. Renaud L, Kishchuk N, Juneau M, Nigam A, Tereault K, Leblanc MC. Implementation and outcomes of $a$ comprehensive worksite health promotion program. Can J Public Health. 2008;99:73-77.

10. Ni Mhurchu C, Aston LM, Jebb SA. Effects of worksite health promotion interventions on employee diets: a systematic review. BMC public health. 2010;10:62.

11. van Dongen JM, Proper KI, van Wier MF, et al. A systematic review of the cost-effectiveness of worksite physical activity and/or nutrition programs. Scand J Work Environ Health. 2012;38:393-408.

12. Eisenson HJ, Binks M. The Duke Diet. New York. Ballantine Books;2007.

13. Eisenson HJ, Westman EC. "Residential Lifestyle Modification Programs for Treatment of Obesity" in Steelman GM and Westman EC. Obesity: Evaluation and Treatment Essentials. New York. Informa Healthcare USA; 2010. 12739.

14. Shapiro JR, Stout AL, Musante GJ. "Structure-size me:" weight and health changes in a four week residential program. Eating behaviors. 2006;7:229-234.

15. Nelius SJ, Heyden S, Hansen JP, Muhlbaier LH, Morris M. Lipoprotein and blood pressure changes during weight reduction at Duke's Dietary Rehabilitation Clinic. Annals of nutrition \& metabolism. 1982;26:384-392.

16. Sjostrom M, Karlsson AB, Kaati G, Yngve A, Green LW, Bygren LO. A four week residential program for primary health care patients to control obesity and related heart risk factors: effective application of principles of learning and lifestyle change. European journal of clinical nutrition. 1999;53 Suppl 2:S72-77.

17. Svetkey LP, Stevens VJ, Brantley PJ, et al. Comparison of strategies for sustaining weight loss: the weight loss maintenance randomized controlled trial. JAMA : the journal of the American Medical Association. 2008;299:1139-1148.

18. Wolever RQ, Webber DM, Meunier JP, Greeson JM, Lausier ER, Gaudet TW. Modifiable disease risk, readiness to change, and psychosocial functioning improve with integrative medicine immersion model. Altern Ther Health Med. 2011;17:38-47.

19. Salinardi TC, Batra $P$, Roberts $S B$, et al. Lifestyle intervention reduces body weight and improves cardiometabolic risk factors in worksites. The American journal of clinical nutrition. 2013;97:667-676.

20. Locatelli SM, Sohn MW, Spring B, Hadi S, Weaver FM. Participant Retention in the Veterans Health Administration's MOVE! Weight management program, 2010. Prev Chronic Dis. 2012;9:E129.

21. Look ARG, Pi-Sunyer $X$, Blackburn $G$, et al. Reduction in weight and cardiovascular disease risk factors in individuals with type 2 diabetes: one-year results of the look AHEAD trial. Diabetes care. 2007;30:1374-1383.
22. Lahiri S, Faghri PD. Cost-effectiveness of a workplacebased incentivized weight loss program. J Occup Environ Med. 2012;54:371-377.

23. Thorndike AN, Healey E, Sonnenberg L, Regan S. Participation and cardiovascular risk reduction in a voluntary worksite nutrition and physical activity program. Preventive medicine. 2011;52:164-166

24. Anderson LM, Quinn TA, Glanz K, et al. The effectiveness of worksite nutrition and physical activity interventions for controlling employee overweight and obesity: a systematic review. American journal of preventive medicine. 2009;37:340-357.

25. Dansinger ML, Tatsioni A, Wong JB, Chung M, Balk EM. Meta-analysis: the effect of dietary counseling for weight loss. Annals of internal medicine. 2007;147:41-50.

26. Appel LJ, Moore TJ, Obarzanek E, et al. A clinical trial of the effects of dietary patterns on blood pressure. DASH Collaborative Research Group. The New England journal of medicine. 1997;336:1117-1124.

27. Svetkey LP, Pollak KI, Yancy WS, Jr., et al. Hypertension improvement project: randomized trial of quality improvement for physicians and lifestyle modification for patients. Hypertension. 2009;54:1226-1233.28.

28. Cook NR, Cutler JA, Obarzanek E, et al. Long term effects of dietary sodium reduction on cardiovascular disease outcomes: observational follow-up of the trials of hypertension prevention [TOHP]. Bmj. 2007;334:885-888.

\section{Acknowledgement}

Funding: The Duke Diet and Fitness Center Duke University Employee Weight Loss Program Pilot Study was supported in part by the Duke Diet and Fitness Center.

The investigators gratefully acknowledge the valuable contributions of the study participants, research and Duke Diet and Fitness Center staff members, including Sofia Rydin-Gray, PhD, Marit Weikel, MA, Janna Fikkan, PhD, Meredith Nahm, $\mathrm{PhD}$, Sandra Houlihan, NP, Nate Reynolds, Caroline Crocker, Gerald K Endress, Dina Lumia, Genise Fowler, and Elisabetta Politi. We would also like to thanks Dr. Prapimporn Chattranukulchai for her assistance organizing the references for this manuscript. 